Tecnología e Innovación

Tecnologia e Inovação

Tecnology and Innovation

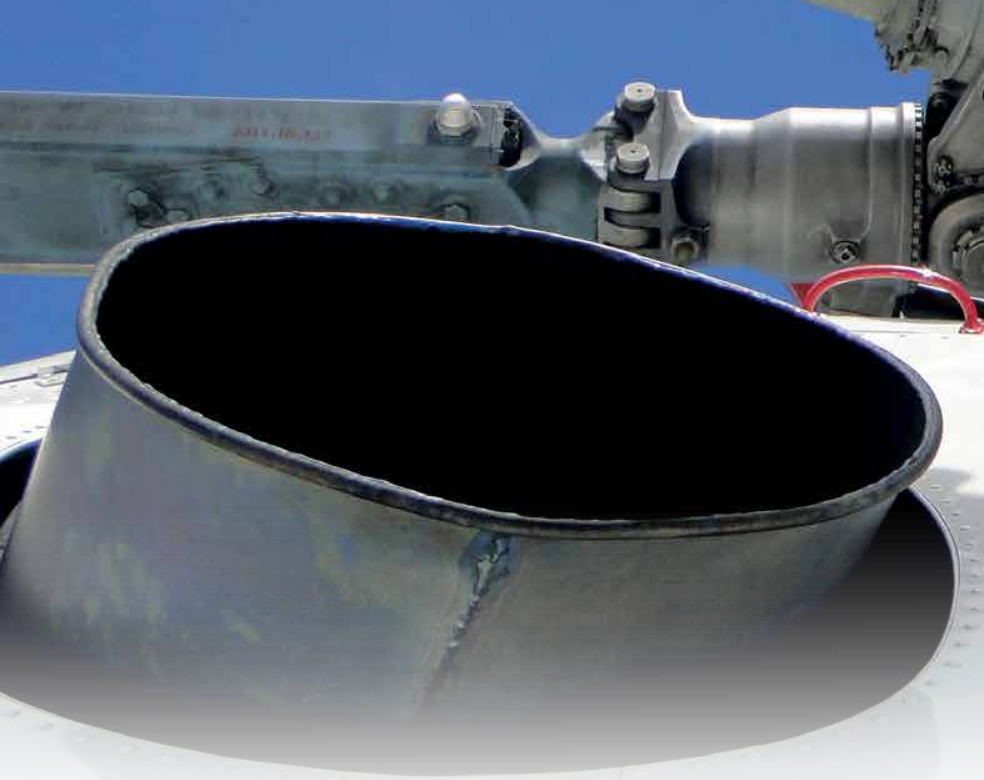

\title{
CocCIÓN DE ALIMENTOS EN BOGOTÁ EMPLEANDO UNA COCINA SOLAR TIPO CAJA ${ }^{1}$
}

COZIMENTO DE ALIMENTOS EM BOGOTÁ USANDO UMA COZINHA SOLAR TIPO CAIXA²

COOKIng FoOd In BOgota Using A BOX COOKER ${ }^{3}$

Silvio Alejandro Jiménez Castellanos ${ }^{4}$, Luis Carlos Rátiva Prieto ${ }^{5}$

Fundación Universitaria Los Libertadores

\section{CIENCIA Y PODER AÉREO}

ISSN 1909-7050 / E- ISSN 2389-2468 / Volumen 10 / Enero-Diciembre de 2015/ Colombia/ Pp. 1-260 Recibido: 22/10/2015

Aprobado evaluador interno: 06/11/2015

Aprobado evaluador externo: 28/11/2015

Doi: http://dx.doi.org/10.18667/cienciaypoderaereo.313 
CIENCIA Y PODER AÉREO | Revista Científica de la Escuela de Postgrados de la Fuerza Aérea Colombiana | Vol. 10 | Enero - Diciembre de 2015

\section{Para citar este artículo:}

Jiménez, S., y Rátiva, L. (2015). Cocción de alimentos en Bogotá empleando una cocina solar tipo caja. Ciencia y Poder Aéreo, 10 (1), 195-208. Doi: http://dx.doi. org/10.18667/cienciaypoderaereo.313

\footnotetext{
'Artículo científico original, derivado del proyecto de investigación: campo transitorio de temperaturas al interior de una cocina solar tipo caja diseñada para las condiciones meteorológicas promedio anuales de Bogotá - Colombia. Trabajo investigativo realizado en el grupo de investigación GIGAD mecánica de la Fundación Universitaria Los Libertadores.
}

${ }^{2}$ Artigo científico original, derivado do projeto de pesquisa: campo de temperatura transiente dentro de uma caixa de cozinh a solar projetado para resistir à taxa média anual de Bogotá - Colômbia. pesquisa realizada no grupo de pesquisa mecânica GIGAD Fundação Universitária Los Libertadores.

${ }^{3}$ Original scientific paper, derived from the research project: transient temperature field inside a solar cooking box designed for the average weather rate of Bogota - Colombia. research conducted in the group of mechanical research GIGAD University Foundation Los Libertadores.

${ }^{4}$ Magíster en docencia de la Universidad de la Salle, Ingeniero mecánico de la Universidad Nacional de Colombia. Docente tiempo completo Fundación Universitaria Los Libertadores, Líder del grupo de investigación GIDAD mecánica, dinamizador del semillero de investigación RETP. Correo electrónico: sajimenezc@libertadores.edu.co

${ }^{5}$ Ingeniero mecánico Fundación Universitaria Los Libertadores, miembro del grupo de investigación GIDAD mecánica, ingeniero de proyectos empresa COLPILAS. Correo electrónico: Icrativap@libertadores.edu.co
Resumen: este artículo tiene como objetivo presentar el campo transitorio de temperaturas de una cocina solar tipo caja básica (sin aditamentos tales como: doble acristalamiento, reflectores internos, reflectores externos, línea de alivio de vapor, entre otros); diseñada para las condiciones meteorológicas promedio anuales de Bogotá - Colombia, con el fin de determinar sí con este dispositivo es posible cocinar en esta ciudad. La metodología utilizada comprendió tres fases: el desarrollo de un modelo físico - matemático del proceso de calentamiento, con base en balances de energía para cada uno de los componentes: vidrio, tapa de recipiente, recipiente, absorbedor, pared y fluido, la realización de un experimento computacional en el software Autodesk Algor Simulation y la comparación de los resultados. La temperatura final del agua obtenida por el modelo físico - matemático fue de $81.85^{\circ} \mathrm{C}$ y la máxima obtenida con la simulación térmica fue de $79.60^{\circ} \mathrm{C}$. De los resultados se puede concluir que en la ciudad de Bogotá D.C no es posible cocinar con un dispositivo de esta clase.

Palabras clave: cocción solar; diseño básico; energía solar térmica; modelamiento matemático; simulación computacional.

Resumo: este artigo tem como objetivo apresentar o campo de temperatura transiente de um fogão solar do tipo de caixa básica (sem anexos, tais como vidros duplos, refletores internos, refletores externos, linha de alívio de vapor, etc.); desenhado para o tempo médio anual em Bogotá - Colômbia, a fim de determinar se é possível cozinhar neste dispositivo nesta cidade. A metodologia consistiu em três fases: o desenvolvimento de um modelo físico -matemático do processo de aquecimento, com base em balanços de energia para cada componente: vidro, tampa do recipiente, recipiente, absorvedor, parede e fluido, a realização de um teste computacional no software Autodesk Algor Simulation e a comparação dos resultados. A temperatura final da água obtido pelo modelo físico - matemático foi $81,85^{\circ} \mathrm{C}$ e a máxima obtida com a simulação térmica era $79,60^{\circ} \mathrm{C}$. A partir dos resultados pode-se concluir que, na cidade de Bogotá D.C. não é possível cozinhar com um dispositivo desta classe.

Palavras-chave: cozimento solar; projeto básico; energia solar; modelagem matemática; simulação de computador.

Abstract: This paper aims to present the transient temperature field of a basic box type solar cooker (without attachments such as double glazing, internal reflectors, external reflectors, steam relief line, etc.) for Bogotá - Colombia climate conditions, in order to determine if this device can cook on its town. The methodology consisted of three main stages: the development of a physical - mathematical model for heating process, based on energy balances for each of its components: glass container lid, container, absorber wall and fluid, the development of a computational experiment in the software Autodesk Algor Simulation and the comparison of the results. The final water temperature obtained by the physical - mathematical model was $81.85^{\circ} \mathrm{C}$, and the maximum obtained with the thermal simulation was $79.60{ }^{\circ} \mathrm{C}$. From the results, it can be concluded that conditions in Bogotá DC are not appropriate to cook with a device of this kind.

Key Words: Basic Design; Computational Simulation; Mathematical Modeling; Solar Cooking; Solar Thermal. 


\section{Introducción}

El uso de la energía se puede clasificar en cuatro sectores: sector doméstico, sector comercial, sector industrial y sector transporte. Entre las aplicaciones de la energía en el sector doméstico está el calentamiento de agua, la calefacción y acondicionamiento de ambientes, la cocción de alimentos, la iluminación, entre otros. Los combustibles que más se utilizan para la cocción de alimentos son la leña y los hidrocarburos (derivados del petróleo, carbón y gas natural). La primera se utiliza en zonas alejadas o de difícil acceso (zonas rurales o zonas marginadas); los segundos, predominantemente en las zonas urbanas.

Además de los ya conocidos efectos del uso de los combustibles fósiles (calentamiento global, contaminación del aire, lluvia ácida, reducción de las fuentes energéticas), hay inconvenientes con el gas natural, debido a los accidentes por inhalación de monóxido de carbono (CO). También hay problemas con la leña debido a la deforestación. Por otro lado, la energía eléctrica no es una alternativa viable ya que en su producción se suele quemar combustibles fósiles, además el montaje y operación del sistema de suministro es costoso.

Otra alternativa es la energía solar ya que es una energía segura, limpia, económica y funcional. El proceso para cocinar alimentos usando energía solar se denomina cocción solar y los dispositivos utilizados se pueden clasificar en cocinas solares directas y cocinas solares indirectas. Dentro de las directas están las de tipo caja, cuyo diseño más básico está compuesto de una cubierta de vidrio, una superficie absorbedora, aislamiento térmico y caja o carcaza (ver Figura 1).

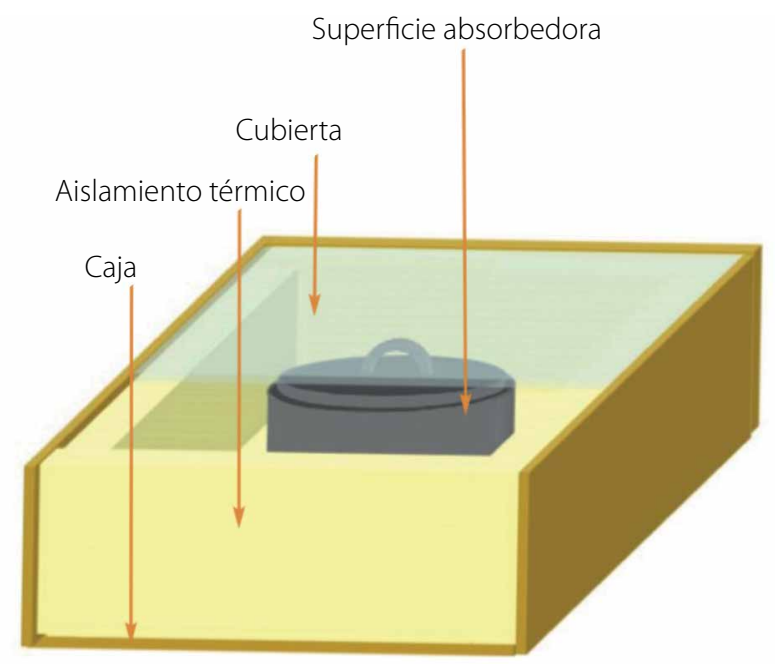

Figura 1. Componentes cocina solar tipo caja básica. (ITC, 2013). Fuente: autores.
Las cocinas tipo caja (también llamadas de acumulación) funcionan con el efecto invernadero: la radiación solar (en gran proporción de onda corta) atraviesa la cubierta de vidrio y calienta la superficie absorbedora (la placa de absorción y el recipiente que contiene el alimento). Esta superficie calienta por convección el aire interior y emite radiación térmica de onda larga que no puede atravesar la cubierta de vidrio, de tal manera que regresa para ser nuevamente absorbida por la superficie de absorción. De esta manera se aumenta la temperatura en el interior de la cocina y la energía térmica acumulada se transfiere a los alimentos contenidos en el recipiente. El proceso continúa hasta que las pérdidas de energía se igualan con la ganancia de energía solar (estado estable). Estos dispositivos generalmente se fabrican con materiales de bajo costo y funcionan $100 \%$ con energía solar, lo cual los hace económicamente asequibles y amigables con el medio ambiente, respectivamente.

Se han realizado varios estudios sobre cocinas solares tipo caja. Aquí se desea destacar los aportes con respecto al diseño hechos por Ekechukwu y Ugwuoke (2003), Macía et al. (2005) y respecto a la determinación del comportamiento térmico, por simulación y/o experimentación, hechos por Schwarzer y Da Silva (2008), Terres, et al. (2008), Passamai (1999), Quiroga et al. (2001) y Chejne et al. (2011). Precisamente este es el único trabajo reportado sobre el análisis del comportamiento térmico de cocinas solares tipo caja en Colombia, específicamente en Medellín.

Como se pudo ver en los antecedentes no hay ningún estudio reportado sobre cocción solar en Bogotá. Por lo tanto, la presente investigación tiene como finalidad establecer si es posible cocinar con energía solar en la ciudad de Bogotá D.C.- Colombia, por medio de la elaboración de un modelo físico-matemático que represente el proceso de calentamiento y de la realización de una simulación térmica computacional de una cocina solar tipo caja básica, diseñada para las condiciones meteorológicas promedio anuales de esta ciudad. Teniendo en cuenta que para que se cocine un alimento este tiene que: sobrepasar los antes de 1.5hr (zona peligosa) para evitar el crecimiento de bacterias y además debe alcanzar los, que corresponde a la temperatura de cocción promedio de los alimentos (Estrada et al, 2003).

También con el proyecto se pretende iniciar estudios más extensos y profundos en energía solar térmica en la ciudad y a la vez fortalecer la investigación en este campo a nivel nacional; ya que la metodología empleada permite extender el análisis del comportamiento del dispositivo a todo el territorio nacional. 
CIENCIA Y PODER AÉREO | Revista Científica de la Escuela de Postgrados de la Fuerza Aérea Colombiana | Vol. 10 | Enero - Diciembre de 2015

El artículo mostrará cómo se hizo el diseño de la cocina, luego el planteamiento y solución del modelo físico matemático que describe el comportamiento térmico del dispositivo y posteriormente la realización del experimento computacional en el programa de simulación.

\section{Método}

Primero se diseñó la cocina con base en el diseño conceptual y los parámetros de diseño (funcionales, energéticos y dimensionales). Posteriormente esta se modeló tridimensionalmente en el software SOLID EGDE ST5. A continuación se realizaron los balances de energía para cada uno de los componentes del dispositivo (vidrio, tapa del recipiente, recipiente, absorbedor, pared y fluido), a partir de los cuales se obtuvo el modelo físico-matemático. Después se simuló el comportamiento térmico de la cocina diseñada en el software Autodesk Algor Simulation. Finalmente se analizaron las curvas de calentamiento de la cocina diseñada, obtenidas mediante: el modelo físico-matemático y la simulación térmica respectivamente.

\section{Diseño de la cocina solar tipo caja Diseño conceptual}

La cocina solar tipo caja de diseño básico de este estudio consta de: a) carcaza: caja de madera que sirve de soporte estructural; b) aislamiento: lana de roca colocada entre la caja interna de aluminio y la caja externa de madera para reducir las pérdidas de calor; c) superficie de absorción: lámina de aluminio de $3 \mathrm{~mm}$ de espesor, cuya geometría se asemeja a la de un cajón y cuya base que en adelante se llamará absorbedor está pintada de negro mate para aumentar la absortividad solar, a la vez que las paredes verticales de esta lámina se denominarán en adelante, pared, d) marco: soporte estructural, ubicado en la parte superior de la cocina, hecho de madera donde se coloca la cubierta y, cuyo mecanismo permite la apertura y el cierre de la cocina, para el ingreso y retirada del recipiente; e) cubierta: lámina de vidrio de $3 \mathrm{~mm}$ de espesor, de geometría rectangular encargada de provocar el efecto invernadero, que en adelante se llamará vidrio y f) recipiente: olla comercial marca Imusa de aluminio, de base plana, pintada de color negro, con una capacidad de almacenamiento de $1.5 \mathrm{~L}$ que equivale a $1.5 \mathrm{~kg}$ de agua, elemento utilizado como estándar para todos los estudios de este tipo, debido a que la gran mayoría de alimentos están compuestos de este elemento.

\section{Parámetros de diseño}

En el diseño de la cocina solar tipo caja para las condiciones meteorológicas promedio anuales de Bogotá Colombia, se definieron y clasificaron los parámetros de diseño en tres categorías, las cuales son: parámetros funcionales (ver Tabla 1), parámetros energéticos (ver Tabla 2) y parámetros dimensionales.

El intervalo de 2,15hr corresponde al tiempo promedio que se recomienda en las guías de cocción solar y se encuentra enmarcado dentro del rango horario de 10:00am a 2:00pm, tiempo en el cual la radiación solar es la más significativa durante el día.

Tabla 1. Parámetros funcionales diseño cocina solar

\begin{tabular}{|c|c|}
\hline Parámetro & Valor \\
\hline Temperatura inicial agua & $14^{\circ} \mathrm{C}$ \\
\hline $\begin{array}{c}\text { Tiempo de cocción } \\
\text { (de ebullición del agua) }\end{array}$ & $2.15 \mathrm{hr}$ \\
\hline $\begin{array}{c}\text { Temperatura final de cocción } \\
\text { (de ebullición del agua) }\end{array}$ & $91.66^{\circ} \mathrm{C}$ \\
\hline $\begin{array}{l}\text { Temperatura máx. esperada } \\
\text { placa de absorción }\end{array}$ & $100^{\circ} \mathrm{C}$ \\
\hline $\begin{array}{l}\text { Temperatura ambiente pro- } \\
\text { medio }\end{array}$ & $14^{\circ} \mathrm{C}(\mathrm{IGAC}, 2010)$ \\
\hline $\begin{array}{c}\text { Carga (masa de agua a ser } \\
\text { hervida) }\end{array}$ & 1.5 kg (Ekechukwu et al.,2003) \\
\hline $\begin{array}{c}\text { Radiación solar promedio } \\
\text { esperada }\end{array}$ & $\begin{array}{l}431 \text { W m² (Hernández et al., } \\
\text { 2010) }\end{array}$ \\
\hline $\begin{array}{c}\text { Fuente: elaboración de los auto } \\
\text { Tabla 2. Parámetros energé }\end{array}$ & icos diseño cocina solar \\
\hline Parámetro & Valor \\
\hline Calor especifico del agua & $\begin{array}{c}4220 \mathrm{~J} \mathrm{Kg}^{-1} \mathrm{oC}^{-1} \\
\text { (Cengel y Boles, 2009) }\end{array}$ \\
\hline $\begin{array}{c}\text { Conductividad térmica } \\
\text { del aislamiento }\end{array}$ & $0.04 \mathrm{~W} \mathrm{~m}^{-1} \mathrm{~K}^{-1}(\mathrm{IVE}, 2013)$ \\
\hline Eficiencia global de la cocina & $\begin{array}{c}35 \% \text { Kg } \\
\text { (Ekechukwu et al.,2003) }\end{array}$ \\
\hline $\begin{array}{c}\text { Pérdidas de calor deseadas } \\
\text { a través de paredes }\end{array}$ & 7\% Kg (Ekechukwu et al.,2003) \\
\hline
\end{tabular}

Los parámetros dimensionales hacen referencia a las dimensiones que va a tener la cocina solar y se obtienen a partir de los funcionales y energéticos establecidos anteriormente. El primero de estos parámetros es el área superficial de la cocina $\mathrm{A}_{\mathrm{s}}$ que se calcula a partir del conocimiento de la eficiencia global, por medio de la Ecuación 1.

$$
\eta=\frac{M C_{p} \Delta T}{I_{a v} t A_{s}}
$$

donde $M$ es la masa del agua en $\mathrm{kg}, \mathrm{C}_{\mathrm{p}}$ es el calor específico del agua a presión constante en $\mathrm{J} \mathrm{Kg}^{-1}{ }^{\circ} \mathrm{C}^{-1}, \Delta \mathrm{T}$ es la diferencia de temperaturas entre la $\mathrm{T}_{\mathrm{i}}$ (temperatura inicial 
del agua) y la $\mathrm{T}_{\mathrm{f}}$ (temperatura final del agua) en ${ }^{\circ} \mathrm{C}$, $\mathrm{I}_{\mathrm{av}}$ es el promedio de la radiación global incidente en $\mathrm{W} \mathrm{m}^{-2}$, $t$ es el tiempo durante el cual se espera que el agua alcance el punto de ebullición en $s$.

El segundo parámetro dimensional es el espesor de la pared del aislamiento $X$ que se obtiene al despejarlo de la Ley de Fourier para la Conducción (ver Ecuación 2).

$$
x=\frac{K A_{L} \Delta T_{P}}{q_{L}}
$$

donde $\mathrm{K}$ es la conductividad térmica del aislante en $W \mathrm{~m}^{-1}{ }^{\circ} \mathrm{C}^{-1}, \mathrm{~A}_{\llcorner}$es el área de pérdidas de calor (fondo más paredes) en $m^{2}, \Delta T_{p}$ es la diferencia de temperaturas entre $T_{p}$ (temperatura de la placa absorbedora) y $T_{a}$ (temperatura ambiente) en ${ }^{\circ} \mathrm{C}, \mathrm{q}_{L}$ es la tasa máxima deseada de pérdidas de calor por las paredes de la cocina en $\mathrm{W} \mathrm{m}^{-2}$.

Reemplazando los parámetros funcionales y energéticos de las Tablas 1 y 2 en las Ecuaciones 1 y 2, se obtienen los parámetros dimensionales de la cocina diseñada (ver Tabla 3).

Tabla 3. Parámetros dimensionales diseño cocina solar

\begin{tabular}{cc}
\hline Parámetro & Valor \\
\hline Área superficial de la cocina & $0,42 \mathrm{~m}^{2}$ \\
\hline Espesor de aislamiento & $0,11 \mathrm{~m}$ \\
\hline Altura interior cocina solar & $0,20 \mathrm{~m}$. \\
\hline
\end{tabular}

Fuente: elaboración de los autores.

El valor de la altura interior de la cocina se obtuvo con base en la altura del recipiente, buscando que dicho valor sea lo más bajo posible, con el fin de reducir el área de pérdidas de calor.

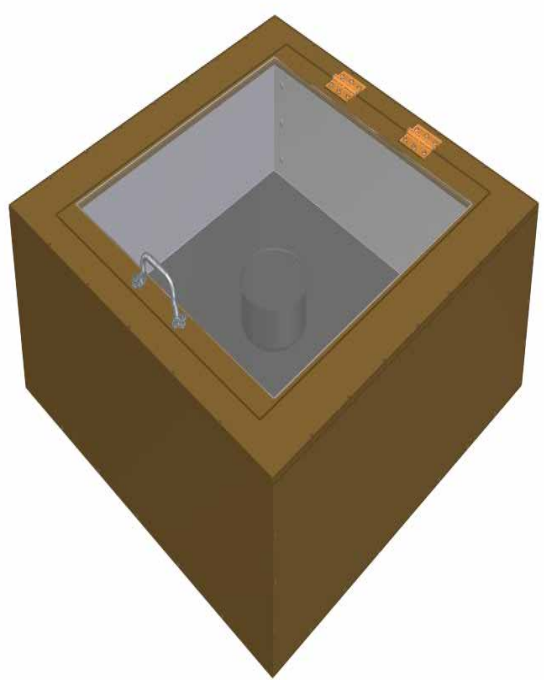

Figura 2. Cocina solar diseñada. Fuente: autores.

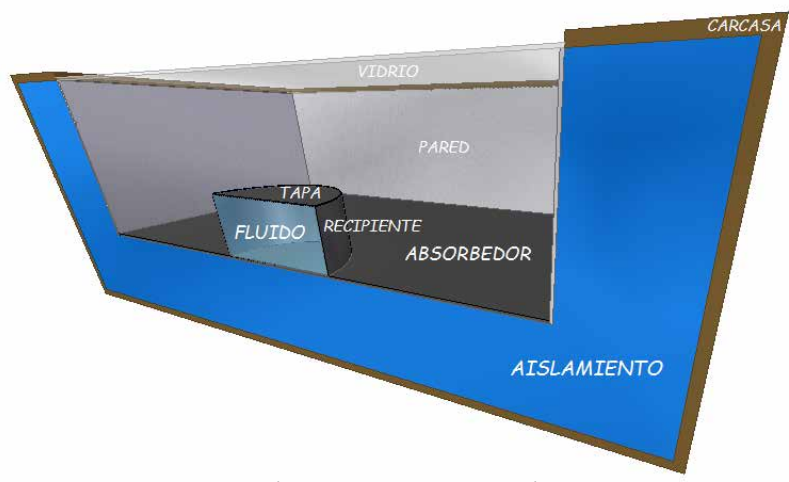

Figura 3. Cocina solar - corte transversal. Fuente: autores.

El prototipo virtual de la cocina, realizado en el software SolidEdge ST5, con base en el diseño conceptual y los parámetros dimensionales se muestra en las Figuras 2 y 3 . En donde se despreciaron las manijas del recipiente y la tapa del mismo, debido a que su sección transversal es muy pequeña en comparación a los demás componentes de la cocina; por lo cual no afectan significativamente en el proceso de calentamiento. Además, se asumió que la tapa y el fondo del recipiente son completamente planas, ya que considerar su geometría real complicaría aún más los cálculos.

\section{Modelo físico - matemático de la cocina \\ Suposiciones y flujos de calor}

El proceso de calentamiento de la cocina diseñada, contempla todos los mecanismos de transferencia de calor: conducción, convección y radiación. Esto ocasiona múltiples flujos de calor en la carcasa, el aislamiento, el vidrio, la tapa de recipiente, el recipiente, el absorbedor, la pared y el fluido. Dada esta gran cantidad de flujos de calor, el análisis del campo de temperaturas de la cocina diseñada es muy complejo y por ello se realizan 23 suposiciones con el fin de simplificar el análisis, sin perder exactitud con respecto al comportamiento real. Las suposiciones son las siguientes:

1. Se desprecia la transferencia de calor por radiación entre la carcasa y los alrededores

\section{$\dot{Q} \underset{R A D . C-E X T .}{N E T}$}

ya que la temperatura de la carcasa se incrementa muy poco, debido a que la transferencia de calor por conducción a través del aislamiento es muy baja, además el material de la carcasa (madera) presenta una baja absortividad a la radiación solar $(a \approx 0.25)$ (Cengel, 2007).

2. Se desprecia la conducción de calor a través del espesor de la carcasa 


\section{$\dot{Q} \underset{\text { CARCASA }}{\operatorname{COND}}$}

ya que la conductividad térmica de su material (madera) es muy baja ( $\left.\mathrm{k}=0.159 \mathrm{~W} \mathrm{~m}^{-1} \mathrm{~K}^{-1}\right)$ (Cengel, 2007). Además se considera como isotérmica dado su bajo espesor $(0.01 \mathrm{~m})$.

3. Se desprecia la convección de calor entre la carcasa y el aire exterior

\section{$\dot{Q} \underset{C-A . E t x}{\operatorname{CONV}}$}

ya que la diferencia de temperatura entre la carcasa y el aire exterior es muy pequeña y debido a que la temperatura de la carcasa se incrementa muy poco. Además se supone la velocidad del viento como "brisa apacible" (3.4-5.4 [ $\left.\mathrm{m} \mathrm{s}^{-1}\right]$ ) (Perales Benito, 2006).

4. Se desprecia la conducción de calor a través del vidrio

$$
\dot{Q} \text { COND }
$$

ya que su conductividad térmica es muy baja ( $k=1$ $\mathrm{W} \mathrm{m}^{-1} \mathrm{~K}^{-1}$ ) (Cengel, 2007). Además se considera como isotérmico dado su bajo espesor $(0.003 \mathrm{~m})$.

5. Se desprecia la conducción de calor a través del aislamiento

\section{$Q \quad$ COND AISLAMIENTO}

ya que su conductividad térmica es muy baja ( $K=0.04$ W $\left.\mathrm{m}^{-1} \mathrm{~K}^{-1}\right)(\mathrm{IVE}, 2013)$.

6. Se desprecian las conducciones de calor a través del absorbedor y la pared,

\section{$\dot{Q} \quad$ COND ABSORBEDOR}

y

$$
\underset{\text { Q COND }}{\dot{P} \text { ARED }}
$$

ya que se consideran como isotérmica dado su bajo espesor $(0.003 \mathrm{~m})$.

7. Se desprecian las irradiaciones $G_{\text {reflejada-absorbedor' }}$ $G_{\text {reflejada-recipiente }}$ y $G_{\text {reffejada-tapa' }}$ ya que debido a su longitud de onda, escapan de la cocina a través de la cubierta de vidrio; además su tasa no es significativa debido al color superficial (negro) que poseen.
8. Se desprecia la irradiación $G_{\text {reflejada-vidrio' }}$ debido a la baja reflectividad de onda corta del material $(\rho \approx 0.06)$.

9. Se desprecian la transferencia de calor por radiación y por conducción entre la carcasa y el soporte de la cocina

$$
\dot{Q}_{R A D}+\dot{Q}_{C O N D_{[C A R C A S A-S O P O R T E]}}
$$

al considerar el fondo de la cocina solar como adiabático.

10. Se desprecian todas las resistencias térmicas de contacto exceptuando: la presente entre el absorbedor y el recipiente, y entre la tapa del recipiente y el fluido, ya que se supone un contacto perfecto en las interfaces de todas las superficies que están en contacto (p.e. interfaz aislamiento - carcaza)

11. Se desprecia la conducción de calor a través de la tapa

\section{$\dot{\mathrm{Q}}_{\mathrm{TOND}}$}

al considerarla como isotérmica dado su bajo espe$\operatorname{sor}(0.001 \mathrm{~m})$.

12. Se desprecian las conducciones de calor a través de la superficie cilíndrica y el fondo del recipiente

\section{$\dot{Q}$ COND}

y

\section{Q COND FONDO DE RECIPIENTE}

al considerarlas como isotérmicas dado su bajo espesor $(0.001 \mathrm{~m})$.

13. Se desprecia la transferencia de calor neta por radiación entre el absorbedor y la superficie lateral vertical de la tapa del recipiente

\section{Q NET RAD. TAPA-ABS.}

debido a que el factor de visión es aproximadamente cero. 
14. Se desprecia la transferencia de calor neta por radiación entre la superficie lateral vertical de la tapa y la pared

\section{Q $\quad$ NET \\ RAD. TAPA-PARED}

y viceversa, debido a que el factor de visión es aproximadamente cero.

15. Se desprecia la transferencia de calor neta del recipiente a la tapa del recipiente

\section{Q NET \\ RAD. RECP.-TAPA}

ya que su disposición geométrica supone un factor de visión igual a 0.

16. Los cálculos de transferencia de calor por radiación se realizarán utilizando la potencia emisiva y la irradiación absorbida.

17. Todas las superficies se consideran grises y difusas, y sus propiedades relativas a la radiación son constantes con la temperatura; con dos valores de emisividad (visible e infrarrojo).

18. Se consideran como superficies opacas: la tapa del recipiente, el recipiente y toda la placa de absorción.

19. En el instante $t=0$ s, se asume que todas las superficies de la cocina solar y el agua se encuentran en equilibrio térmico con la temperatura ambiente promedio de Bogotá (14 $\mathrm{C} \approx 287.15 \mathrm{~K}$.) (IGAC, 2010).

20. Se asume que el absorbedor estará a una temperatura mayor que el recipiente, debido a que tiene un área mayor y por lo tanto capta más energía.

21. El fluido (agua) se encuentra en contacto perfecto con el recipiente y la tapa del mismo.

22. La transferencia de calor del recipiente hacia el fluido es mediante el mecanismo de convección.

23. Se asume que toda la $G_{\text {reflejada-pared }}$ llega al absorbedor.

Tomando en cuenta las anteriores suposiciones, los flujos que se tendrán en cuenta son los que se muestran en las Figuras 4 y 5.

\section{Ecuaciones gobernantes}

Con base en las suposiciones mencionadas en el aparte anterior, se realizan balances de energía para el vidrio, la tapa del recipiente, el recipiente, el absorbedor, la pared y el fluido; que en total relacionan 23 flujos de calor. Con estos balances se obtuvo finalmente un modelo matemático, conformado por seis ecuaciones diferenciales ordinarias (EDO) y ocho incógnitas. Para reducir dos incógnitas, se plantean dos ecuaciones algebraicas adicionales para la temperatura del cielo T_c y para la del aire en el interior de la cocina T_int (ver Ecuaciones 9 y 10). El modelo permite predecir la temperatura, en kelvin, de todos los componentes en cada instante de tiempo:

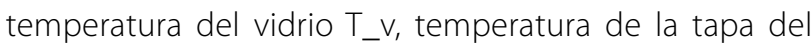
recipiente $T_{-} t$, temperatura del recipiente $T_{r}$ temperatura del absorbedor T_a, temperatura de la pared T_p y temperatura del fluido (agua) T_f. Las ecuaciones que conforman el modelo son las siguientes:

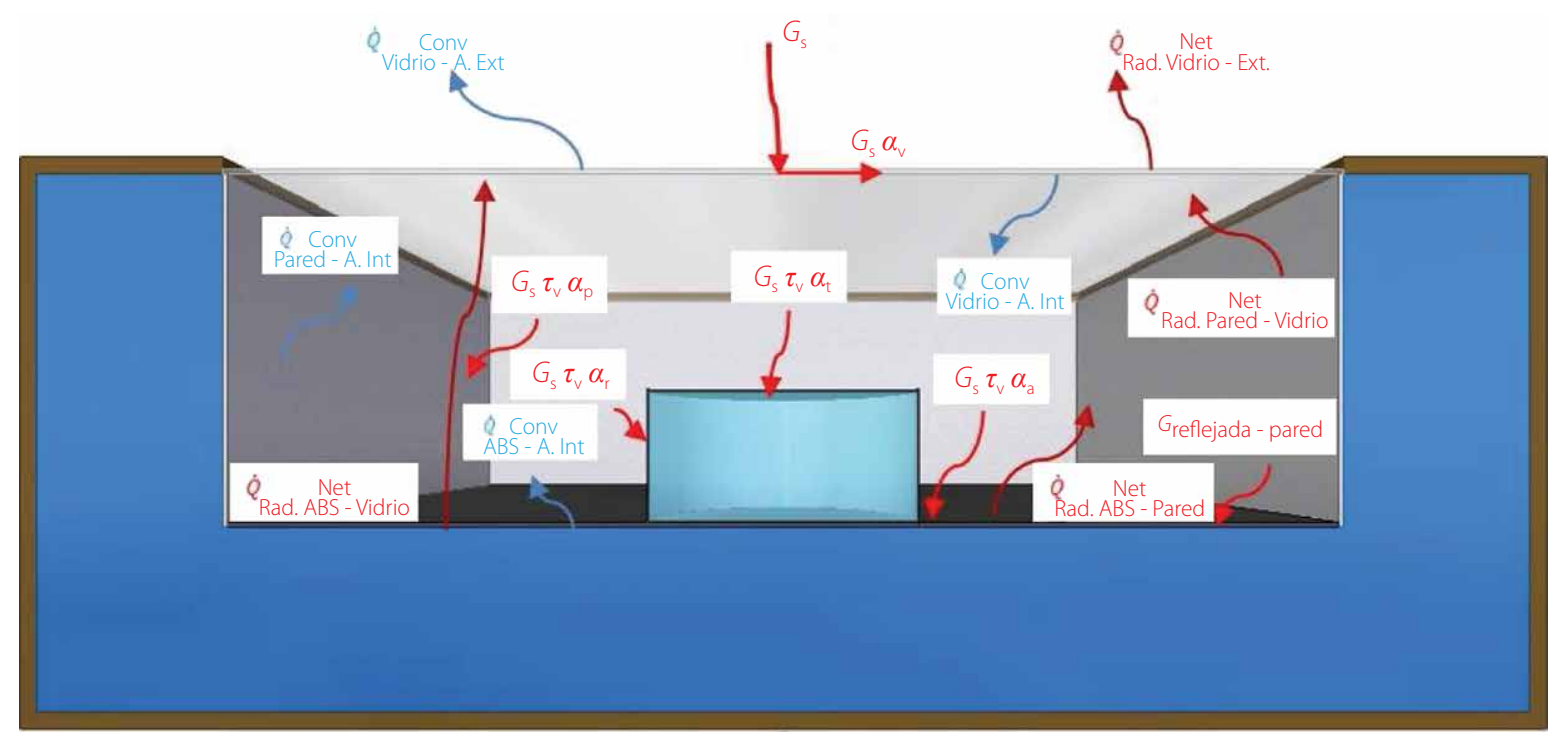

Figura 4. Flujos de calor cocina solar. Fuente: autores. 


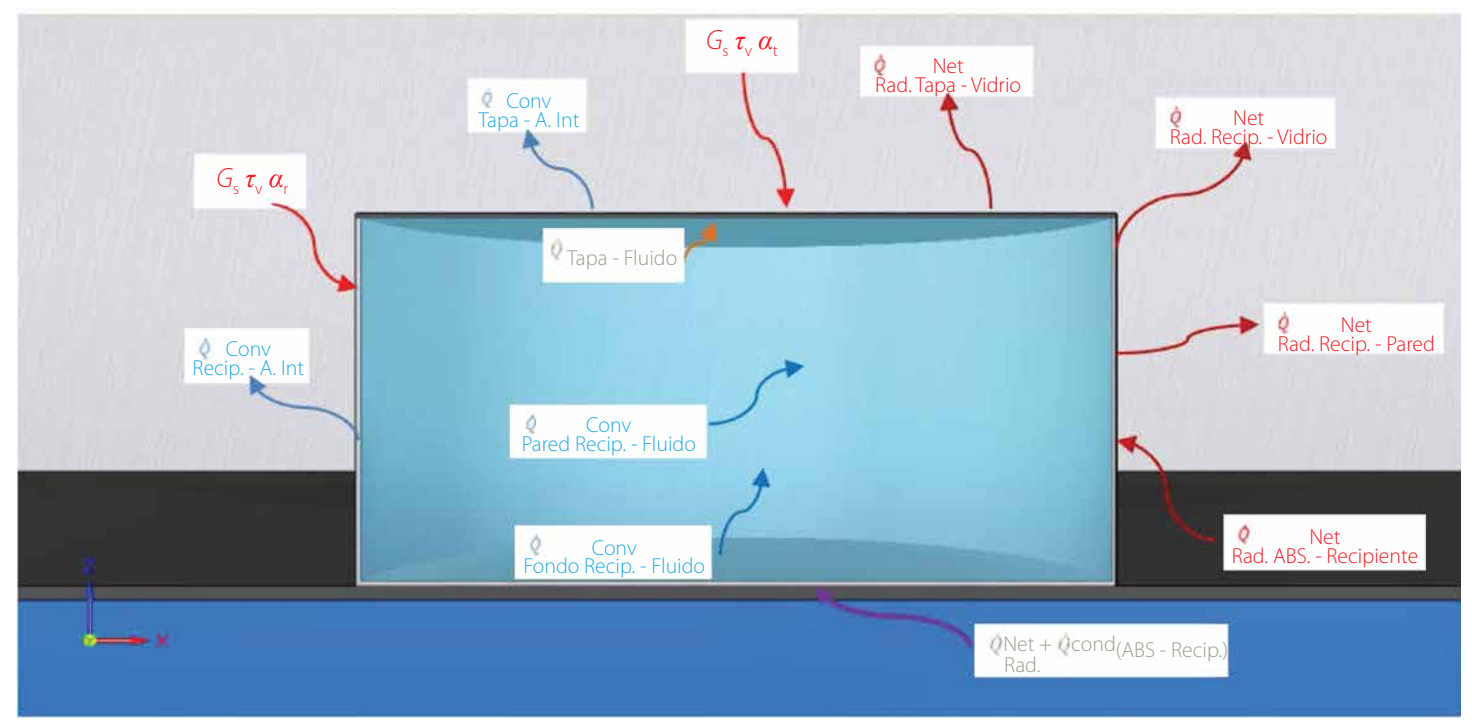

Figura 5. Detalle flujos de calor recipiente y fluido. Fuente: autores.

Balance de energía del vidrio.

$$
\frac{d T_{v}}{d t}=\frac{\dot{Q}_{1}+\dot{Q}_{2}+\dot{Q}_{3}+\dot{Q}_{4}+\dot{Q}_{5}+\dot{Q}_{6}-\dot{Q}_{7}-\dot{Q}_{8}}{m_{v} * C p_{v}}\left[\frac{K}{s}\right]
$$

$\dot{Q}_{1}=A_{v} * G_{s} * \alpha_{v}[\mathrm{~W}]$ Flujo de calor por radiación solar adsorbido por el vidrio.

$\dot{\mathrm{Q}}_{2}=\mathrm{A}_{\mathrm{t}}{ }^{*} \sigma^{*} \varepsilon_{\mathrm{t}}{ }^{*} \mathrm{~F}_{\mathrm{tv}}\left(\mathrm{T}_{\mathrm{t}}^{4}-\mathrm{T}_{\mathrm{v}}^{4}\right)[\mathrm{W}]$ Flujo de calor por radiación de la tapa del recipiente hacia el vidrio.

$\dot{\mathrm{Q}}_{3}=\mathrm{A}_{\mathrm{r}}{ }^{*} \sigma^{*} \varepsilon_{\mathrm{r}}{ }^{*} \mathrm{~F}_{\mathrm{rv}}\left(\mathrm{T}_{\mathrm{r}}^{4}-\mathrm{T}_{\mathrm{v}}^{4}\right)[\mathrm{W}]$ Flujo de calor por radiación del recipiente hacia el vidrio.

$\dot{\mathrm{Q}}_{4}=\mathrm{A}_{\mathrm{a}}{ }^{*} \sigma^{*} \varepsilon_{\mathrm{a}} * \mathrm{~F}_{\mathrm{av}}\left(\mathrm{T}_{\mathrm{a}}^{4}-\mathrm{T}_{\mathrm{v}}^{4}\right)[\mathrm{W}]$ Flujo de calor por radiación del absorbedor hacia el vidrio.

$\dot{\mathrm{Q}}_{5}=\mathrm{A}_{\mathrm{p}}{ }^{*} \sigma^{*} \varepsilon_{\mathrm{p}}{ }^{*} \mathrm{~F}_{\mathrm{pv}}\left(\mathrm{T}_{\mathrm{p}}^{4}-\mathrm{T}_{\mathrm{v}}^{4}\right)[\mathrm{W}]$ Flujo de calor por radiación de la pared hacia el vidrio.

$\dot{\mathrm{Q}}_{6}=\mathrm{A}_{\mathrm{v}}{ }^{*} \mathrm{~h}_{\mathrm{v} \text {-int }}\left(\mathrm{T}_{\mathrm{int}}-\mathrm{T}_{\mathrm{v}}\right)[\mathrm{W}]$ Flujo de calor por convección del interior hacia el vidrio.

$\dot{\mathrm{Q}}_{7}=\mathrm{A}_{\mathrm{v}}{ }^{*} \sigma^{*} \varepsilon_{\mathrm{v}}{ }^{*} \mathrm{~F}_{\mathrm{vc}}\left(\mathrm{T}_{\mathrm{v}}^{4}-\mathrm{T}_{\mathrm{c}}^{4}\right)[\mathrm{W}]$ Flujo de calor por radiación del vidrio hacia el cielo.

$\dot{\mathrm{Q}}_{8}=\mathrm{A}_{\mathrm{v}}{ }^{*} \mathrm{~h}_{\mathrm{v}-\mathrm{amb}}\left(\mathrm{T}_{\mathrm{v}}-\mathrm{T}_{\mathrm{amb}}\right)[\mathrm{W}]$ Flujo de calor por convección del vidrio hacia el ambiente.

Balance de energía en la tapa del recipiente.

$$
\frac{d T_{t}}{d t}=\frac{-\dot{Q}_{2}+\dot{Q}_{9}-\dot{Q}_{10}-\dot{Q}_{11}}{m_{t} * C p_{t}}\left[\frac{K}{s}\right]
$$

$\dot{Q}_{9}=A_{t} * G_{s} * \tau_{v} * \alpha_{t}[\mathrm{~W}]$ Flujo de calor por radiación solar adsorbido por la tapa del recipiente.

$\dot{Q}_{10}=A_{t-f} * \frac{T_{t}-T_{f}}{R_{t h-t f}}[\mathrm{~W}]$ Flujo de calor de la tapa del recipiente hacia el fluido.

$$
\dot{Q}_{11}=A_{t} * h_{t-i n t}\left(T_{t}-T_{\text {int }}\right)[\mathrm{W}] \text { Flujo de calor por convección }
$$

de la tapa del recipiente hacia el interior. 
Balance de energía del recipiente.

$$
\frac{d T_{r}}{d t}=\frac{-\dot{Q}_{3}+\dot{Q}_{12}+\dot{Q}_{13}+\dot{Q}_{14}-\dot{Q}_{15}-\dot{Q}_{16}-\dot{Q}_{17}}{m_{r} * C p_{r}}\left[\frac{K}{s}\right]
$$

$\dot{Q}_{12}=A_{r} * G_{s} * \tau_{v} * \alpha_{r}[\mathrm{~W}]$ Flujo de calor por radiación solar adsorbido por el recipiente.

$$
\dot{Q}_{13}=A_{a} * \sigma * \varepsilon_{a} * F_{a r}\left(T_{a}^{4}-T_{r}^{4}\right)[\mathrm{W}] \text { Flujo de calor por radiación }
$$

del absorbedor hacia el recipiente.

$$
\begin{gathered}
\dot{Q}_{14}=A_{a-r} * \frac{T_{a}-T_{r}}{R_{t h-a r}}[\mathrm{~W}] \text { Flujo de calor del absorbedor hacia el recipiente. } \\
\dot{Q}_{15}=A_{r} * \sigma * \varepsilon_{r} * F_{r p}\left(T_{r}^{4}-T_{p}^{4}\right)[\mathrm{W}] \text { Flujo de calor por radiación }
\end{gathered}
$$

del recipiente hacia las paredes.

$$
\begin{gathered}
\dot{Q}_{16}=A_{r} * h_{r-i n t}\left(T_{r}-T_{\text {int }}\right)[\mathrm{W}] \text { Flujo de calor por convección del recipiente hacia el interior. } \\
\dot{Q}_{17}=A_{r-f} * h_{r-f}\left(T_{r}-T_{f}\right)[\mathrm{W}] \text { Flujo de calor por convección del recipiente hacia el fluido. }
\end{gathered}
$$

Balance de energía del absorbedor.

$$
\frac{d T_{a}}{d t}=\frac{-\dot{Q}_{4}-\dot{Q}_{13}-\dot{Q}_{14}+\dot{Q}_{18}+\dot{Q}_{19}-\dot{Q}_{20}-\dot{Q}_{21}}{m_{a} * C p_{a}}\left[\frac{K}{s}\right]
$$

$\dot{Q}_{18}=A_{a} * G_{s} * \tau_{v} * \alpha_{a}[\mathrm{~W}]$ Flujo de calor por radiación solar adsorbido por el absorbedor.

$\dot{Q}_{19}=G_{s} * \tau_{v} * \rho_{p} * A_{a} * \alpha_{a}[\mathrm{~W}]$ Flujo de calor por radiación solar reflejada por las paredes.

$$
\dot{Q}_{20}=A_{a} * \sigma * \varepsilon_{a} * F_{a p}\left(T_{a}^{4}-T_{p}^{4}\right)[\mathrm{W}] \text { Flujo de calor por radiación }
$$

del absorbedor hacia la pared.

$\dot{Q}_{21}=A_{a} * h_{a-i n t}\left(T_{a}-T_{\text {int }}\right)$ [W] Flujo de calor por convección del absorbedor hacia el interior.

Balance de energía del recipiente.

$$
\begin{gathered}
\qquad \frac{d T_{p}}{d t}=\frac{-\dot{Q}_{5}+\dot{Q}_{15}+\dot{Q}_{20}+\dot{Q}_{22}-\dot{Q}_{23}}{m_{p} * C p_{p}}\left[\frac{K}{s}\right] \\
\dot{Q}_{22}=A_{p} * G_{s} * \tau_{v} * \alpha_{p}[\mathrm{~W}] \text { Flujo de calor por radiación solar adsorbido por la pared. } \\
\dot{Q}_{23}=A_{p} * h_{p-i n t}\left(T_{p}-T_{\text {int }}\right)[\mathrm{W}] \text { Flujo de calor por convección de la pared hacia el interior. } \\
\text { Balance de energía del fluido } \quad \text { Temperatura del cielo } \quad \text { Temperatura aire interior de la cocina } \\
\frac{d T_{f}}{d t}=\frac{\dot{Q}_{17}-\dot{Q}_{10}}{m_{f} * C p_{f}}\left[\frac{K}{s}\right] \text { (8) } \quad T_{c}=0,0552 * T_{a m b}^{1,5}\left[{ }^{\circ} \mathrm{C}\right](9) \quad T_{i n t}=\frac{T_{v}+T_{t}+T_{r}+T_{a}+T_{p}}{5}\left[{ }^{\circ} \mathrm{C}\right] \text { (10) }
\end{gathered}
$$


CIENCIA Y PODER AÉREO | Revista Científica de la Escuela de Postgrados de la Fuerza Aérea Colombiana | Vol. 10 | Enero - Diciembre de 2015

\section{Datos de entrada}

La Tabla 4 relaciona datos utilizados para determinar los valores de las condiciones iniciales del modelo físico - matemático. Los factores de visión se consideraron difusos.

Tabla 4. Datos entrada solución modelo físico - matemático

\begin{tabular}{|c|c|c|}
\hline Parámetro & Símbolo & Valor \\
\hline Área neta Absorbedor & $\mathrm{A}_{\mathrm{a}}$ & $0.397 \mathrm{~m}^{2}$ \\
\hline Área contacto Absorbedor - Recipiente & $\mathrm{A}_{\mathrm{ar}}$ & $0.020 \mathrm{~m}^{2}$ \\
\hline Área Pared & $A_{P}$ & $0.520 \mathrm{~m}^{2}$ \\
\hline Área cilíndrica del Recipiente & $\mathrm{A}_{\mathrm{r}-\text { cilindrica }}$ & $0.039 \mathrm{~m}^{2}$ \\
\hline Área contacto Recipiente - Fluido & $A_{r-f}$ & $0.058 \mathrm{~m}^{2}$ \\
\hline Área de la Tapa del recipiente & $\mathrm{A}_{\mathrm{t}}$ & $0.02268 \mathrm{~m}^{2}$ \\
\hline Área contacto Tapa del recipiente - Fluido & $A_{t-f}$ & $0.0196 \mathrm{~m}^{2}$ \\
\hline Área Vidrio & $A_{v}$ & $0.423 \mathrm{~m}^{2}$ \\
\hline Calor específico Absorbedor & $\mathrm{Cp}_{\mathrm{a}}$ & $880 \mathrm{~J} \mathrm{~kg}^{-1} \mathrm{~K}^{-1}$ (Estrada et al., 2003) \\
\hline Calor específico Fluido (agua) & $\mathrm{Cp}_{\mathrm{f}}$ & $4220 \mathrm{~J} \mathrm{~kg}^{-1} \mathrm{~K}^{-1}$ (Cengel y Boles, 2009) \\
\hline Calor específico Pared & $\mathrm{Cp}_{\mathrm{p}}$ & $880 \mathrm{~J} \mathrm{~kg}^{-1} \mathrm{~K}^{-1}$ \\
\hline Calor específico Recipiente & $\mathrm{Cp}_{\mathrm{r}}$ & $880 \mathrm{~J} \mathrm{~kg}^{-1} \mathrm{~K}^{-1}$ \\
\hline Calor específico Tapa de recipiente & $\mathrm{Cp}_{\mathrm{t}}$ & $880 \mathrm{~J} \mathrm{~kg}^{-1} \mathrm{~K}^{-1}$ \\
\hline Calor específico Vidrio & $\mathrm{Cp}_{\mathrm{v}}$ & $745 \mathrm{~J} \mathrm{~kg}^{-1} \mathrm{~K}^{-1}$ (Estrada et al., 2003) \\
\hline Coeficiente de convección Absorbedor - Interior & $\mathrm{h}_{\mathrm{a}-\mathrm{int}}$ & $4 \mathrm{~W} \mathrm{~m}^{-2} \mathrm{~K}^{-1}$ \\
\hline Coeficiente de convección Pared - Interior & $\mathrm{h}_{\mathrm{p} \text {-int }}$ & $4.4 \mathrm{~W} \mathrm{~m}^{-2} \mathrm{~K}^{-1}$ \\
\hline Coeficiente de convección Recipiente - Fluido & $\mathrm{h}_{\mathrm{r}-\mathrm{f}}$ & $4 \mathrm{~W} \mathrm{~m}^{-2} \mathrm{~K}^{-1}$ (Terrés et al., 2008) \\
\hline Coeficiente de convección Recipiente - Interior & $\mathrm{h}_{\mathrm{r} \text {-int }}$ & 4.4 $\mathrm{W} \mathrm{m}^{-2} \mathrm{~K}^{-1}$ (Terrés et al., 2008) \\
\hline $\begin{array}{c}\text { Coeficiente de convección Tapa de recipiente - } \\
\text { Interior }\end{array}$ & $\mathrm{h}_{\mathrm{t} \text {-int }}$ & $4 \mathrm{~W} \mathrm{~m}^{-2} \mathrm{~K}^{-1}$ (Terrés et al., 2008) \\
\hline Coeficiente de convección Vidrio - Ambiente & $\mathrm{h}_{\mathrm{v}-\mathrm{amb}}$ & $13.30 \mathrm{~W} \mathrm{~m}^{-2} \mathrm{~K}^{-1}$ (Terrés et al., 2008) \\
\hline Coeficiente de convección Vidrio - Interior & $\mathrm{h}_{\mathrm{v} \text {-int }}$ & 4.4 $\mathrm{W} \mathrm{m}^{-2} \mathrm{~K}^{-1}$ (Terrés et al., 2008) \\
\hline Constante de Stefan -Boltzman & $\sigma$ & $5.67 \times 10^{8} \mathrm{~W} \mathrm{~m}^{-2} \mathrm{~K}^{-4}$ (Terrés et al., 2008) \\
\hline Factor de visión Absorbedor hacia Pared & $\mathrm{F}_{\mathrm{ap}}$ & 0.4 \\
\hline Factor de visión Tapa de recipiente hacia Vidrio & $\mathrm{F}_{\mathrm{tv}}$ & 1 \\
\hline Factor de visión Recipiente hacia Vidrio & $\mathrm{F}_{\mathrm{rv}}$ & 0.026 \\
\hline Factor de visión Absorbedor hacia Vidrio & $\mathrm{F}_{\mathrm{av}}$ & 0.589 \\
\hline Factor de visión Pared hacia Vidrio & $\mathrm{F}_{\mathrm{pv}}$ & 0.33 \\
\hline Factor de visión Absorbedor hacia Recipiente & $\mathrm{F}_{\mathrm{ar}}$ & 0.011 \\
\hline Factor de visión Recipiente hacia Pared & $\mathrm{F}_{\mathrm{rp}}$ & 0.86 \\
\hline Factor de visión Vidrio hacia Cielo & $\mathrm{F}_{\mathrm{vc}}$ & 1 \\
\hline Masa Absorbedor & $\mathrm{m}_{\mathrm{a}}$ & $3.21 \mathrm{~kg}$ \\
\hline Masa Fluido & $\mathrm{m}_{\mathrm{f}}$ & $1.5 \mathrm{~kg}$ \\
\hline Masa Pared & $\mathrm{m}_{\mathrm{p}}$ & $4.21 \mathrm{~kg}$ \\
\hline Masa Recipiente & $\mathrm{m}_{\mathrm{r}}$ & $0.2352 \mathrm{~kg}$ \\
\hline Masa Tapa de recipiente & $\mathrm{m}_{\mathrm{t}}$ & $0.0448 \mathrm{~kg}$ \\
\hline Masa Vidrio & $\mathrm{m}_{\mathrm{v}}$ & $3.43 \mathrm{~kg}$ \\
\hline Absortividad Absorbedor - onda corta & $\alpha_{\mathrm{a}}$ & 0.90 (Estrada et al., 2003) \\
\hline Absortividad Pared - onda corta & $\alpha_{\mathrm{p}}$ & 0.15 (Cengel, 2007) \\
\hline Absortividad Recipiente - onda corta & $\alpha_{\mathrm{r}}$ & 0.90 (Estrada et al., 2003) \\
\hline
\end{tabular}




\begin{tabular}{|c|c|c|}
\hline Absortividad Tapa de recipiente - onda corta & $\alpha_{t}$ & 0.90 \\
\hline Absortividad Vidrio - onda corta & $\alpha_{\mathrm{v}}$ & 0.15 (Cengel, 2007) \\
\hline Emisividad Absorbedor - onda larga & $\varepsilon_{\mathrm{a}}$ & 0.80 (Estrada et al., 2003) \\
\hline Emisividad Pared - onda larga & $\varepsilon_{\mathrm{p}}$ & 0.05 (Cengel, 2007) \\
\hline Emisividad Recipiente - onda larga & $\varepsilon_{\mathrm{r}}$ & 0.80 (Estrada et al., 2003) \\
\hline Emisividad Tapa de recipiente - onda larga & $\varepsilon_{\mathrm{t}}$ & 0.80 \\
\hline Emisividad Vidrio - onda larga & $\varepsilon_{\mathrm{v}}$ & 0.80 (Estrada et al., 2003) \\
\hline Reflectividad Pared & $\rho_{\mathrm{p}}$ & 0.85 \\
\hline Transmisividad Vidrio a la radiación solar & $\tau_{\mathrm{v}}$ & 0.79 (Cengel, 2007) \\
\hline Radiación solar global & $\mathrm{G}_{\mathrm{s}}$ & $431 \mathrm{~W} \mathrm{~m}^{-2}$ (Hernández et al., 2010) \\
\hline $\begin{array}{l}\text { Resistencia térmica de contacto Absorbedor - } \\
\text { Recipiente }\end{array}$ & $\mathrm{R}_{\text {th-ar }}$ & $0.00088 \mathrm{~m}^{2}{ }^{\circ} \mathrm{C} \mathrm{W}^{-2}$ \\
\hline $\begin{array}{l}\text { Resistencia térmica de contacto Tapa de recipiente - } \\
\text { Fluido }\end{array}$ & $\mathrm{R}_{\mathrm{th}-\mathrm{tf}}$ & $0.00088 \mathrm{~m}^{2}{ }^{\circ} \mathrm{C} \mathrm{W}^{-2}$ \\
\hline Temperatura inicial Absorbedor & $\mathrm{T}_{\mathrm{a}}$ & $14{ }^{\circ} \mathrm{C} \approx 287.15 \mathrm{~K}(\mathrm{GAC}, 2002)$ \\
\hline Temperatura inicial Ambiente & $\mathrm{T}_{\mathrm{amb}}$ & $14^{\circ} \mathrm{C} \approx 287.15 \mathrm{~K}$ \\
\hline Temperatura inicial Cielo & $\mathrm{T}_{\mathrm{c}}$ & $2,89^{\circ} \mathrm{C} \approx 276.04 \mathrm{~K}$ \\
\hline Temperatura inicial Fluido & $\mathrm{T}_{\mathrm{f}}$ & $14^{\circ} \mathrm{C} \approx 287.15 \mathrm{~K}$ \\
\hline Temperatura inicial Interior & $\mathrm{T}_{\text {int }}$ & $14{ }^{\circ} \mathrm{C} \approx 287.15 \mathrm{~K}$ \\
\hline Temperatura inicial Pared & $\mathrm{T}_{\mathrm{p}}$ & $14^{\circ} \mathrm{C} \approx 287.15 \mathrm{~K}$ \\
\hline Temperatura inicial Recipiente & $\mathrm{T}_{\mathrm{r}}$ & $14{ }^{\circ} \mathrm{C} \approx 287.15 \mathrm{~K}$ \\
\hline Temperatura inicial Tapa de recipiente & $\mathrm{T}_{\mathrm{t}}$ & $14{ }^{\circ} \mathrm{C} \approx 287.15 \mathrm{~K}$ \\
\hline Temperatura inicial Vidrio & $\mathrm{T}_{\mathrm{v}}$ & $14{ }^{\circ} \mathrm{C} \approx 287.15 \mathrm{~K}$ \\
\hline
\end{tabular}

Fuente: elaboración de los autores.

\section{Experimento computacional}

El experimento computacional que simula el calentamiento de la cocina solar diseñada se realizó aplicando el módulo de transferencia de calor del programa de simulación Autodesk Algor Simulation, en un modelo tridimensional de elementos finitos.

Se modelaron seis elementos básicos de la cocina: absorbedor, pared, vidrio, recipiente, tapa de recipiente y el fluido (modelada como un cilindro sólido de agua), debido a que la superficie exterior de la placa metálica se supone adiabática. Para el enmallado se utilizaron elementos finitos tipo "brick"y"thetraedal" (Ver Figura 6).

Los mecanismos de transferencia de calor: conducción, convección y radiación se incluyeron en el modelo como condiciones de frontera (cargas y restricciones térmicas). Los coeficientes de transferencia de calor se tomaron de la Tabla IV. Para los cálculos de transferencia de calor por radiación se utilizó una herramienta especial llamada "Body - Body radiation".
La simulación se realizó utilizando un análisis de estado transitorio para un período de 2.15 horas (7740 s). El intervalo de tiempo se dividió de tal manera para que la temperatura fuera calculada cada 600s (10 minutos). La condición inicial para todos los componentes es una temperatura uniforme y demás datos de entrada para la simulación, también se tomaron de la Tabla 4.

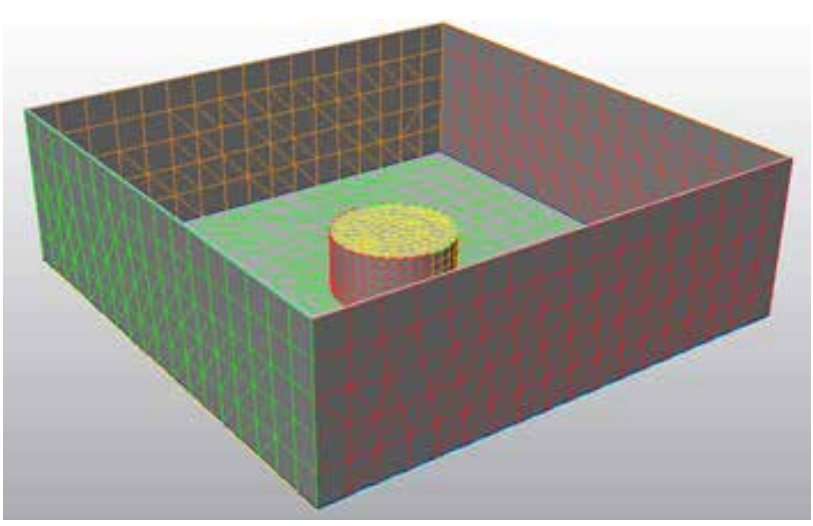

Figura 6. Enmallado del modelo tridimensional. Fuente: autores. 


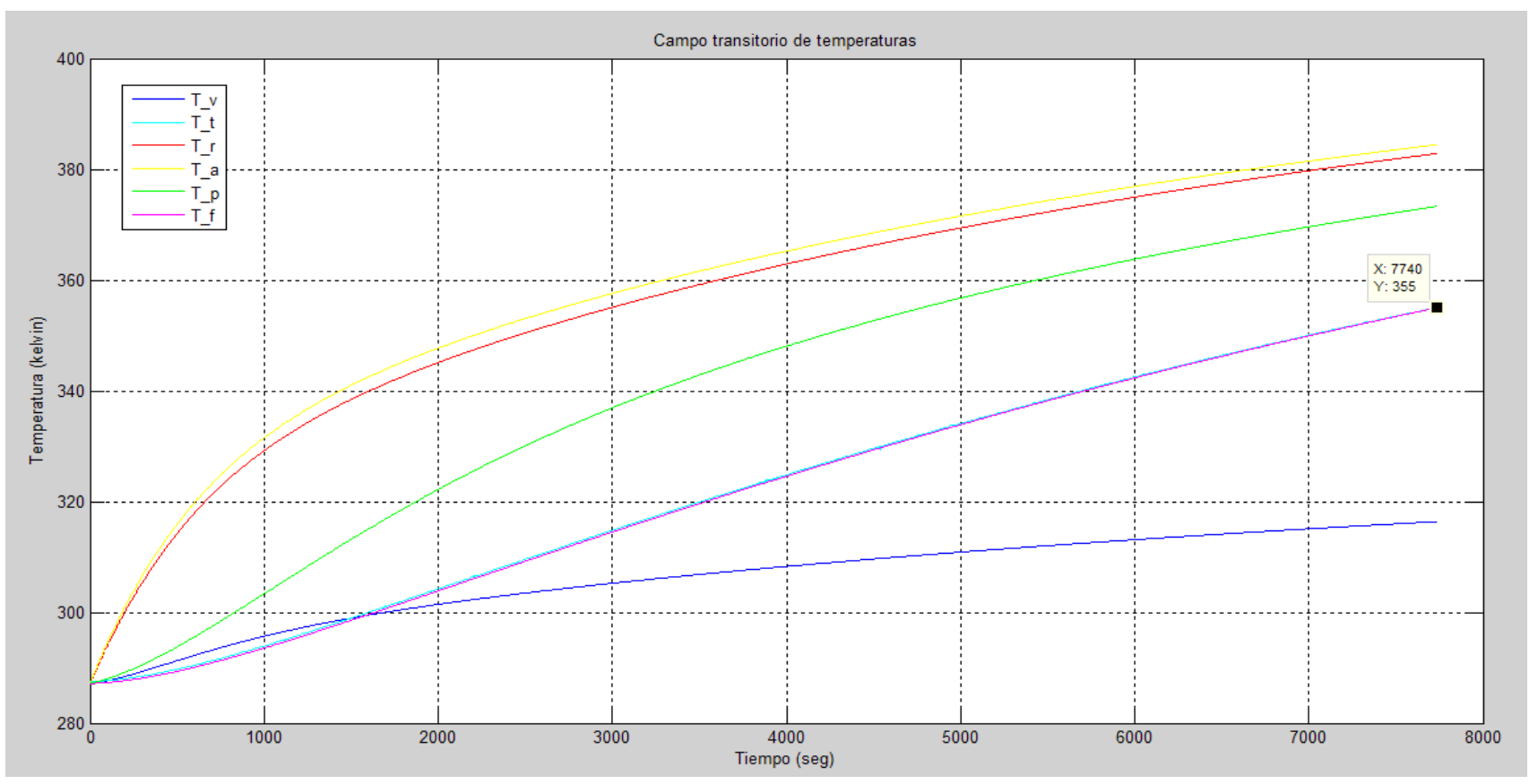

Figura 7. Historial de temperaturas cocina solar diseñada. Fuente: autores.

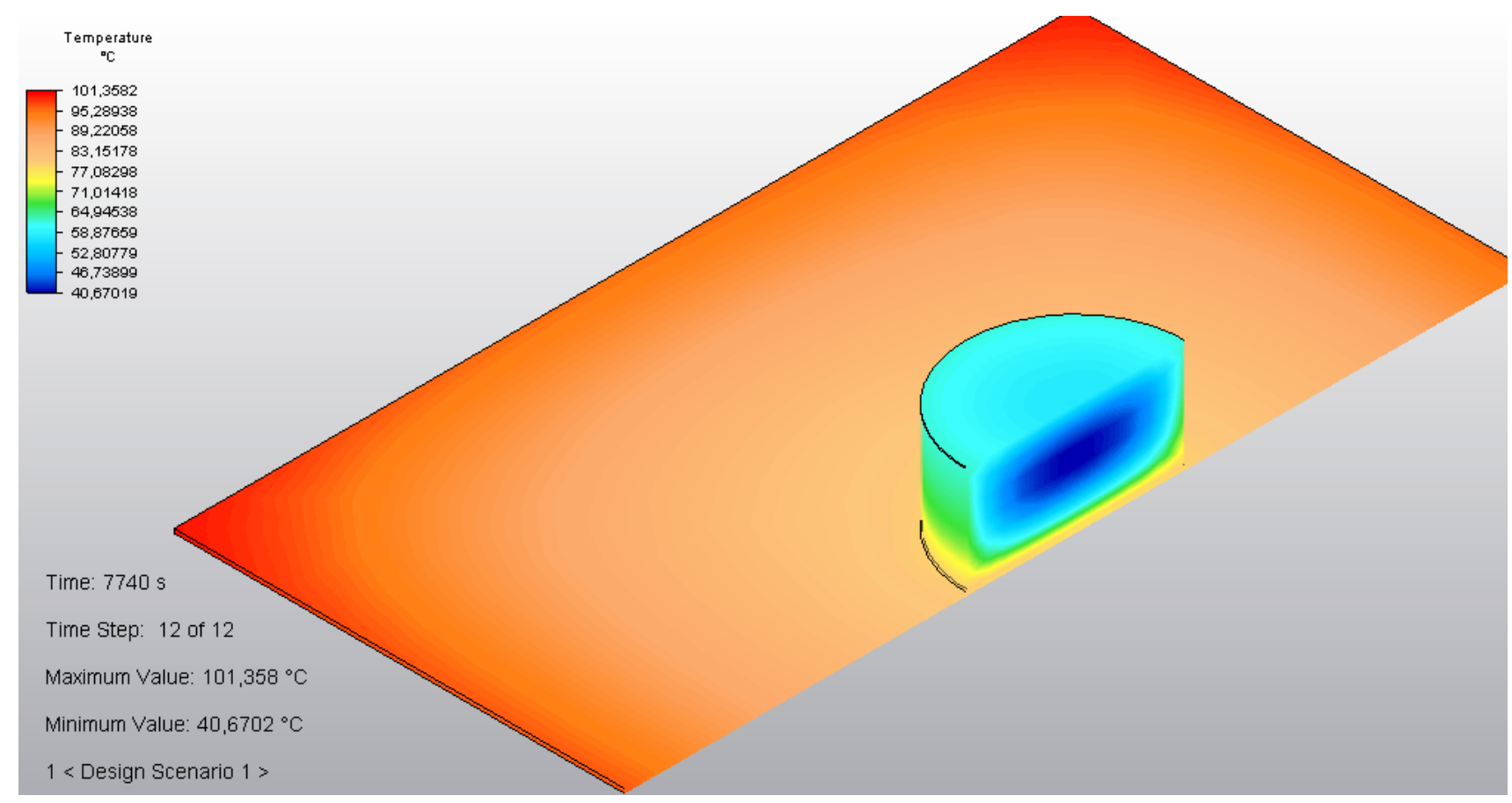

Figura 8. Campo de temperaturas - 2.15 horas de proceso de calentamiento: placa absorción - agua. Fuente: autores.

\section{Resultados y discusión}

\section{Solución modelo físico - matemático}

El modelo físico - matemático resultante está conformado por seis EDO`s (ecuaciones diferenciales ordinarias) y dos ecuaciones algebraicas, con 58 condiciones iniciales.
Dado que estas ecuaciones son muy complejas de resolver por métodos analíticos, se emplean métodos numéricos, para su solución. Dicha solución se llevó acabo en el software MATLAB 7.6.0 (R2008a), el cual cuenta con ocho solucionadores de EDO`s, de las cuales se seleccionó el solucionador "ode113". 
La Figura 7 muestra la historia de temperaturas para los componentes de la cocina, donde $T_{v}$ es la temperatura de la del vidrio, $T_{t}$ es la temperatura de la tapa del recipiente, $T_{r}$ la temperatura del recipiente, $T_{\mathrm{a}}$ temperatura del absorbedor, $T_{p}$ temperatura de la pared y $T_{f}$ temperatura del fluido (agua). Las temperaturas están dadas en grados Kelvin.

La primera observación importante, aunque obvia que se puede hacer de esta Figura es que todos los componentes se están calentando. Para la placa de absorción, recipiente, pared metálica y vidrio es claro el comportamiento exponencial de la temperatura, con valores más altos para los dos primeros en todo momento, como era de esperarse por estar pintados de negro mate. También, se observa que durante los primeros 1600 s, la temperatura del agua y de la tapa está ligeramente por debajo de la de la cubierta, lo cual puede deberse a que el calor específico del vidrio es menor que el del agua y el aluminio. Después de este instante de tiempo, la temperatura del agua y tapa supera a la del vidrio, como era de esperarse.

De la Figura 7, también se puede inferir que la temperatura del agua después 1.5 horas (5400 s) fue de $337.4 \mathrm{~K} \approx$ $64.25^{\circ} \mathrm{C}$ y en 2.15 horas $\left(7740 \mathrm{~s}\right.$ ) fue de $355 \mathrm{~K} \approx 81.85^{\circ} \mathrm{C}$.

Respecto a la velocidades de calentamiento (dT/dt) todas son positivas. Sin embargo, para la placa, el recipiente y el vidrio esta cantidad va disminuyendo con el transcurrir del tiempo, siendo más notable este fenómeno en los dos primeros que en último. Para la pared metálica la velocidad de calentamiento solo empieza a disminuir aproximadamente a partir de los 800 s. Lo mismo ocurre para la tapa del recipiente y el fluido (agua), pero alrededor de los $1600 \mathrm{~s}$.

Finalmente, también observar que la evolución de temperatura del fluido y de la tapa del recipiente es casi igual, ya que sus curvas de calentamiento prácticamente se superponen.

\section{Solución por experimento computacional}

La Figura 8 muestra el campo escalar transitorio de temperaturas de placa de absorción y del agua, pasados 7740 s (2.15 horas) del proceso de calentamiento. La cubierta y la placa metálica se ocultaron para facilitaron la visualización. Se observa que la temperatura del agua está entre $79.6^{\circ} \mathrm{C}$, en la parte inferior (en la base cerca de la placa de absorción) y $40.6^{\circ} \mathrm{C}$ en la parte central del cilindro de agua. También, se observa que la parte superior del cilindro de agua está a una temperatura más alta que la del centro.

\section{Conclusiones}

Después de haber desarrollado el diseño, el modelo físico - matemático y la simulación térmica de una cocina solar tipo caja de diseño básico, se pueden inferir que no es posible cocinar con este dispositivo bajo condiciones meteorológicas promedio anuales de la ciudad de Bogotá D.C. Sin embargo, se recomienda realizar un estudio con la misma cocina utilizada en este estudio pero adicionándole aditamentos tales reflectores internos y reflectores externos, ya que estos permitirían que una mayor cantidad de energía llegue al recipiente (olla) y por lo tanto también al fluido (agua).

El modelo físico - matemático desarrollado describe de manera coherente el calentamiento de todos los componentes de la cocina. Por ejemplo, los componentes más opacos y de color oscuro a la radiación son los que más se calientan (absorbedor, recipiente y tapa de recipiente), con respecto a los semi-transparentes a la radiación (vidrio) y a los que tienen una alta reflectividad (pared).

Por medio de la simulación térmica se confirma que el recipiente recibe la mayor tasa de transferencia de calor desde el absorbedor.

El campo de temperaturas obtenido mediante el modelo físico - matemático y la simulación térmica, demuestran que el agua es el componente que más se demora en empezarse a calentar, esto debido a su inercia térmica producto de su alto calor especifico.

Se recomienda dar continuación al presente proyecto, al realizar una investigación en donde se construya la cocina solar diseñada y se realice el respectivo experimento físico con el fin de contrastar esos resultados con los obtenidos en el presente proyecto. Posteriormente desarrollar otro proyecto de investigación en el cual la cocina solar diseñada se integre a una comunidad vulnerable de la ciudad de Bogotá D.C, para establecer como se da la interacción entre dispositivo y usuario.

\section{Agradecimientos}

Los autores agradecen al ingeniero Camilo Mejía, ingeniero mecánico de la Universidad Nacional de Colombia, ya que con su apoyo se pudo realizar esta investigación y escribir este artículo.

\section{Referencias}

Cengel, Y. A. (2007).Transferencia de calor por radiación. En Transferencia de calor y masa ( $3^{\circ}$ ed.) (p. 663 a 709). México D. F, Mc. Graw Hill. 
CIENCIA Y PODER AÉREO | Revista Científica de la Escuela de Postgrados de la Fuerza Aérea Colombiana | Vol. 10 | Enero - Diciembre de 2015

Cengel, Y. A. (2007). Apéndice 1: Tablas y diagramas de propiedades sistema internacional. En Transferencia de calory masa $\left(3^{\circ}\right.$ ed.) (p. 841 a 867). México D.F.: Mc. Graw Hill.

Cengel, Y. A., y Boles M.A. (2009). Apéndice A tablas de propiedades, figuras y diagramas. En Termodinámica. (p. 912). México D.F: Mc Graw Hill.

Chejne, F., Macía, A., Estrada, D., Velásquez, H. I., \& Londoño, C. (2011).Radiation model for predicting temperature evolution in solar cooker. Revista Dyna, 166, 68-74

Ekechukwu, O. V., \& Ugwuoke, N. T. (2003). Design and measured performance of a plane reflector augmented box-type solar-energy cooker. Renewable Energy, 28, 1935 -1952. Recuperado el 20 de marzo de 2010 de la base de datos Science Direct.

Estrada, D. A., y Macía, A. F. (2003). Diseño, construcción y puesta en operación de una cocina solar para la preparación de productos alimenticios. Trabajo de grado para optar al título de ingeniero mecánico. Universidad Nacional de Colombia sede Medellín, Medellín, Colombia.

Hernández, J.; Sáenz, E., y Vallejo, W. A. (2010). Estudio del Recurso Solar en la Ciudad de Bogotá para el Diseño de Sistemas Fotovoltaicos Interconectados Residenciales. Revista Colombiana de Física, 42, (2), 161-165.

Instituto Geográfico Agustín Codazzi - IGAC (2002). Atlas de Colombia. (5a Edición). Bogotá: Imprenta Nacional de Colombia.

Instituto Tecnológico de Canarias - ITC. (2007). Guía didáctica de energía solar - hornos solares. Recuperado el 30 de septiembre de 2013. [En línea] Disponible en http://www.itccanarias. org/web/difusion/recursos_didacticos/Energia/Documentacion/

Instituto Valenciano de la Edificación - IVE. (s.f.) Productos y materiales: propiedades de aislantes térmicos para rehabilitación energética. Recuperado 30 de septiembre de 2013. [En línea] Disponible en http://www.five.es/descargas/archivos/P1_ portada.pdf

Macía, A. F., et al. (2005). Metodología para el diseño conceptual de cocinas solares. Revista Dyna, 146, 65-88.

Passamai, V. (1999). Simulación del comportamiento térmico de una cocina solar Parte II. Avances en Energías Renovables y Medio Ambiente, 3, (2).

Perales Benito, T. (2006). Energía eólica. En Guía del instalador de energías renovables ( $1^{\circ}$ ed.) (p. 89). México D.F.: Limusa.

Quiroga, M., Saravia, L. R., y Cadena, C. (2001). Aplicación del programa sceptre para la simulación de una cocina solar tipo caja. Avances en Energías Renovables y Medio Ambiente, 5, 7-12.

Schwarzer, K. \& Da Silva, M. (2008). Characterization and design methods of solar cookers. Solar energy, 82, 157-163. Recuperado el 20 de marzo de 2010 de la base de datos Science Direct.
Terrés H, Ortega A, Gordon M., Morales, J. R. y Lizardi, A. (2008). Evaluación de reflectores internos en una estufa solar de tipo caja. Científica, 12, (4), 175-183. 